\title{
émulations
}

\section{L'internationalisation du goût activités marchandes des immigrés grecs en Belgique et en Australie}

\section{Katerina Seraïdari}

Émulations - Revue de sciences sociales

2018, n²6, « Repenser la dichotomie 'national vs international' »

\section{Article disponible à l'adresse suivante}

https://ojs.uclouvain.be/index.php/emulations/article/view/seraidari

\section{Pour citer cet article}

Seraïdari Katerina, "L'internationalisation du goût activités marchandes des immigrés grecs en Belgique et en Australie ». Émulations, №26, pp. 105-116. Mise en ligne le 15 décembre 2018.

DOI : 10.14428/emulations.026.08

Distribution électronique : Université catholique de Louvain (Belgique) : ojs.uclouvain.be

(C) Cet article est mis à disposition selon les termes de la Licence Creative Commons Attribution, Pas d'Utilisation Commerciale 4.0 International. http://creativecommons.org/licenses/by-nc/4.0/

Éditeur : Émulations - Revue de sciences sociales / Presses universitaires de Louvain https://ojs.uclouvain.be/index.php/emulations

ISSN électronique : 1784-5734

PUL PRESSES UNIVERSITAIRES 


\title{
L'internationalisation du goût
}

\author{
Activités marchandes des immigrés grecs en \\ Belgique et en Australie
}

\begin{abstract}
Katerina Seraïdari ${ }^{1}$
[Résumé] L'article analyse le rôle que les immigrés grecs ont joué en Australie et en Belgique sur la croissance des échanges alimentaires, le rapprochement des comportements de consommation et l'élargissement du répertoire culinaire. Dans les deux cas, l'internationalisation apparaît comme une stratégie marchande permettant à un commerce fondé par des immigrés d'être viable. La juxtaposition de ces deux terrains révèle également que l'internationalisation se présente sous deux formes tant complémentaires que contradictoires : une forme qui maintient les barrières nationales et qui ethnicise, tout en s'appuyant sur l'exoticisation ; et une forme qui tend à refuser la spécificité nationale et à neutraliser tout ce qui apparaissait jusqu'alors exotique.

Mots-clés : immigration ; internationalisation ; alimentation ; Grèce ; Australie ; Belgique.

[Abstract] This article analyzes the role that Greek immigrants played in Australia and Belgium concerning the augmentation of food exchanges, the alignment of consumption habits and the opening of the culinary repertoire. In both cases, internationalization constitutes a commercial strategy assuring viability to a business funded by immigrants. The juxtaposition of these two fieldworks also shows that internationalization takes two complementary but also contradictory forms: a form that maintains national frontiers and ethnicizes as much as it exoticizes; and a form that refuses national specificity and neutralizes what looked until then exotic.
\end{abstract}

Keywords: immigration; internationalization; food; Greece; Australia; Belgium.

L'étude des pratiques commerçantes dans le domaine de l'alimentation permet de saisir une partie des processus de «mondialisation par le bas » auxquels les immigrés participent (Portes, 1999), ainsi que les voies par lesquelles l'internationalisation du goût a progressé au cours du XX $\mathrm{XX}^{\mathrm{e}}$ siècle. Deux mouvements migratoires seront au centre de cet article : celui des Grecs vers l'Australie dès le début du XX siècle, et celui des Grecs vers la Belgique dans les années 1950. Alors que la Belgique et l'Australie ont toutes deux connus des vagues d'immigration importantes après la Deuxième Guerre mondiale, l'Australie est pour sa part un pays fondé par l'immigration, au même titre que les États-Unis ou le Canada.

L'immigration grecque après 1950 est réglementée par un certain nombre de conventions, comme celle qui est signée avec l'Australie en 1952, et avec la Belgique en 1957. Après la Deuxième Guerre mondiale et jusque dans les années 1970, l'Australie est le

${ }^{1}$ Membre associée du Centre d'Anthropologie Sociale de Toulouse (LISST). 
pays non européen qui reçoit le plus grand nombre d'immigrés grecs. Ceux-ci ont dominé le domaine de l'alimentation depuis les premières décennies du $\mathrm{XX}^{\mathrm{e}}$ siècle jusque dans les années 1970 en ouvrant des magasins et des cafés qui ne vendaient alors aucun produit grec. Les spécialités grecques quittent leurs cuisines domestiques et deviennent accessibles à la clientèle australienne seulement à la fin des années 1970 (Alexakis, Janiszweski, 2016: 21) : un descendant de ces commerçants grecs caractérise d'ailleurs les spécialités grecques qui n'ont été que tardivement proposées aux Australiens comme « notre nourriture secrète » (Ibid. : 129). Un autre informateur, arrivé en Australie en 1956 et propriétaire d'un certain nombre de cafés et de restaurants, avoue que les Grecs de sa génération n'ont pas eu le courage d'introduire la nourriture grecque dans leur pays d'accueil (Ibid. : 238).

Quant aux Grecs de Belgique, une fois qu'ils quittent les mines et les usines belges ${ }^{2}$, ils s'aventurent dans le domaine de l'alimentation. Ils se vantent même d'avoir appris aux Belges à consommer des produits grecs - souvent caractérisés comme « méditerranéens » afin d'attirer une clientèle plus large (Seraïdari, 2012a). Néanmoins, depuis quelques années, la plupart des épiceries encore tenues par des Grecs à Bruxelles n'offrent presque plus de produits grecs que l'on peut facilement trouver dans les supermarchés et les supérettes. Ces magasins ont donc progressivement perdu leur caractère ethnique.

Dans les deux cas, l'internationalisation du goût apparaît comme une ressource assurant la viabilité et la compétitivité des commerces fondés par des Grecs dans leur pays d'accueil. L'internationalisation dont il sera question dans cet article ne constitue la conséquence ni d'une politique mise en place par l'État ${ }^{3}$ ni d'une politique entrepreneuriale planifiée par des détenteurs de capitaux importants. Ces immigrés cherchaient, tout simplement, le modèle économique et les opportunités marchandes leur permettant d'améliorer leur statut social et leur condition économique. C'est pour cette raison qu'une internationalisation de ce type se présente comme un processus désordonné et spontané.

D’un point de vue méthodologique, la présentation de l'activité commerciale des Grecs en Australie est basée sur le livre d'Alexakis et Janiszweski (2016) qui est le fruit d'une recherche exhaustive conduite depuis 1982 et qui constitue le travail le plus documenté sur les activités commerciales dans le domaine de l'alimentation en ce qui concerne l'ensemble de la diaspora grecque. En revanche, l'analyse concernant les

\footnotetext{
${ }^{2}$ C'est en 1955 que les premiers Grecs commencent à arriver sur le territoire belge pour travailler dans les mines. Ayant achevé les années exigées dans les charbonnages, certains d'entre eux ont pu obtenir un permis de travail de catégorie $A$ et s'orienter vers l'industrie ou des métiers moins pénibles.

${ }^{3}$ Tel que ce fut par exemple le cas en France où les ambassades et les événements gastronomiques qui y sont organisés ont joué « un rôle essentiel dans la reconnaissance à l'étranger d'une singularité du luxe alimentaire français » (Marcihac, $2012: 67$ ).
} 
Grecs de Bruxelles privilégiera les données ethnographiques que j’ai recueillies entre 2009 et $2015^{4}$.

Les activités marchandes des immigrés grecs révèlent, comme nous le verrons, l'importance de l'immigration en tant que « phénomène mondialisant » tant au niveau économique que culturel. Même s'il s'agit de stratégies individuelles et familiales, le processus de transformation qu'elles déclenchent acquiert une dimension collective, du fait de l'émulation au sein du groupe immigré, mais aussi de ses effets cumulatifs sur la société d'accueil. Le but sera ainsi d'examiner quel rôle les immigrés grecs ont joué en Australie et en Belgique sur la croissance des échanges alimentaires, le rapprochement des comportements de consommation et l'élargissement du répertoire culinaire.

La mise en parallèle de ces deux cas révèle que seule l'ethnicisation de l'offre parvient à internationaliser le paysage gastronomique d'une ville ou d'un pays. L'altérité et l'hétérogénéité nourrissent l'internationalisation : il faut donc des commerces grecs, italiens, chinois, indiens ou américains ${ }^{5}$. Sans ethnicisation, point d'internationalisation (du moins, dans un premier temps) ; l'une présuppose l'autre. Ce qui est « ethniquement exotique » peut être banalisé seulement quand les effets d'intégration ont atteint un seuil élevé. En somme, il faut progressivement faire exister de nouvelles pratiques alimentaires, puis les rendre incontournables, avant que cette diversification soit neutralisée par une homogénéisation généralisée. Dans ce cadre évolutif, l'ethnicisation ne constitue pas un processus régressif ; bien au contraire, elle apparaît comme une étape nécessaire pour décloisonner le domaine de l’alimentation.

\section{Commerçants grecs en Australie, acteurs de l'américanisation}

Le « Café grec » en Australie est tenu par des Grecs, mais vend des produits qui n’ont rien à voir avec le pays d'origine. C'est à travers ces commerces populaires, qui ont connu leur apogée entre le milieu des années 1930 et la fin des années 1960, que les immigrés grecs ont pu imposer des modèles de consommation venant des États-Unis (Alexakis, Janiszewski, 2016:12). Si ceux-ci sont devenus des vecteurs de changement,

\footnotetext{
${ }^{4}$ Dans le cadre du projet « Quand deux insularités socio-culturelles se côtoient au cœur de l'Europe : le rapport à l'intégration des communautés grecque et turque de Bruxelles et leurs relations mutuelles », financé par l'IRSIB (Profil A, Convention «Brains (back) to Brussels »), sous la supervision d'Ural Manço (Facultés universitaires Saint-Louis, 2009-2010); et dans le cadre du projet «Building social relationships through commercial transactions: Turkish grocers and their multicultural clientele in Brussels » en tant que junior EURIAS fellow au Vlaams Academisch Centrum de Bruxelles (2012-2013).

${ }^{5}$ Comme nous le verrons, l'américanisation, qui caractérise les initiatives commerciales des Grecs en Australie résulte, d'une part, d'un mixage de traditions nationales et d'innovations technologiques à l'intérieur des États-Unis et, d'autre part, d'un modèle à visée universelle. Même si l'américanisation est souvent considérée comme un synonyme de la mondialisation, je la considère ici comme une forme d'ethnicisation. De ce point de vue, toutes les formes d'ethnicisation ne se valent pas, certaines d'entre elles étant hégémoniques.
} 
c'est parce qu'ils avaient déjà été familiarisés avec les innovations américaines : les premières décennies du $\mathrm{XX}^{e}$ siècle connaissent un mouvement migratoire qui amène les Grecs des États-Unis vers l'Australie (Ibid.: 14). Même ceux qui viennent directement de Grèce ont des parents et des amis aux États-Unis ; les contacts et les échanges entre ces groupes d'immigrés sont fréquents. Une fois installés en Australie, ces "Grecs itinérants »- tel que l'un des informateurs de ces deux chercheurs les nomme - ouvrent des cafés, des «bars américains » et inventent même une nouvelle formule, celle du « milk bar » où ils vendent des sodas et contribuent à rendre les milkshakes extrêmement populaires, surtout après 1932 (Ibid. : 15). La circulation permanente des personnes et des concepts marchands entre le continent américain et le continent australien influe aussi sur les pratiques de consommation. Si le milkshake est un produit d'origine américaine que les Cafés grecs adoptent, adaptent aux données locales et popularisent en Australie, le stationnement de soldats américains en Australie pendant la Deuxième Guerre mondiale et leur retour aux États-Unis après la fin du conflit relancera la mode des milkshakes (Ibid. : 121) : ce modèle de consommation, une fois redéfini en Australie, sera réimporté aux États-Unis et rencontrera un succès jamais égalé jusque-là, les soldats devenant dans ce cas des agents de la «mondialisation par le bas ».

Des fontaines de soda sont installées dans les magasins que les Grecs d'Australie tiennent depuis les années 1910 ; ces équipements viennent directement des États-Unis, principalement de Chicago (Ibid. : 36, 100). Toutefois, la fabrication et la diffusion par la grande distribution des boissons en canette et en bouteille mettent fin, dès le milieu des années 1970, à cette mode des fontaines de soda (Ibid.: 226). Depuis les années 1970, l'apparition des premiers fast-foods américains - le premier McDonald's ouvre à Sydney en 1971 (Ibid. : 57) - et l'expansion des supermarchés mettent à mal les petites entreprises familiales grecques.

À noter par ailleurs que les commerces que ces immigrés ouvrent en Australie sont intimement liés à l'industrie du cinéma, puisqu'ils accueillent les clients avant la projection des films, pendant la pause et après leur sortie, mais aussi parce qu'ils prolongent le rêve américain en dehors des salles de projection. Selon un autre informateur d'Alexakis et de Janiszewski, «le cinéma leur promettait Hollywood. Le café continuait l'illusion » (Ibid.: 17). Déjà dans les années 1930, les Cafés grecs et les cinémas sont reliés par « une relation de travail symbiotique » (Ibid. : 88). L’arrivée de la télévision et l'abandon partiel de ce type de loisir affecteront également les Cafés grecs qui voient leur clientèle diminuer.

Pour les Grecs d'Australie, l'intégration dans la société d'accueil est longtemps passée par l'effacement des références renvoyant au pays d'origine. En revanche, pour les Grecs de Bruxelles, la mise en avant de leur spécificité nationale a facilité leur réussite économique. 


\section{Commerçants grecs de Bruxelles, acteurs de l'internationalisation}

Dans son livre sur la commune bruxelloise de Saint-Gilles, Alphonse Debouverie (1992 : 190) parle de l'internationalisation du secteur de l'alimentation et de « quelques concentrations marquantes : italienne dans le quartier Louise, grecque dans le quartier du Midi et à la place Bethléem, orientale rue de Moscou, espagnole et portugaise dans le centre de la commune ». Il publie également une photographie de la place Bethléem en 1975, où l'on voit une épicerie grecque avec une enseigne Coca-Cola (Ibid. : 122). Si certaines communes bruxelloises peuvent se vanter de pouvoir proposer une offre culinaire internationale dès les années 1970, d'autres devront attendre encore quelques décennies avant de connaître un développement similaire, dans la mesure où cette diversification de l'offre est liée aux espaces d'habitation et d'activité commerciale de différents groupes immigrés.

Les commerçants grecs de Bruxelles se présentent comme ceux qui ont réussi à introduire dans le paysage alimentaire belge des produits grecs jusqu'alors inconnus, et à les y ancrer de façon durable. Selon un grossiste grec qui a ouvert son magasin dans un quartier commerçant de Sainte-Catherine en janvier 1983, mais qui collaborait avec les épiciers grecs de Bruxelles depuis 1969, « dans les années 1970, l’État grec aidait ceux qui faisaient des exportations, ils recevaient alors une prime de $10 \%$. Parce que nous étions des missionnaires [ierapostoloi]. Nous, les exportateurs, mais aussi les immigrés. C'est l'immigré qui a amené ici la civilisation grecque et les produits grecs » (entretien, mardi 18 novembre 2014).

En effet, ce n'est qu'après l'entrée de la Grèce dans la Communauté économique européenne en 1981 que cette politique étatique favorisant les exportations au sein de l'Europe a pris fin. Pays sans colonies et sans développement industriel pouvant attirer une main-d'œuvre étrangère, la Grèce avait jusque-là une économie non seulement fermée, mais aussi dépendante des fonds envoyés par les immigrés grecs. L'ouverture du marché et la mondialisation de son secteur alimentaire sont des phénomènes nouveaux liés à l'adhésion de 1981 (Papageorgiou, 2010).

Le récit du grossiste grec, qui concorde avec d'autres témoignages que j'ai recueillis, érige l'immigré en porteur d'une civilisation qui se répand au gré des déplacements de ceux qui la représentent. Il révèle ainsi la valeur que les acteurs de la « mondialisation par le bas » assignent à leur propre contribution. Fiers de leur réussite commerciale, ceux-ci aiment endosser le rôle de « missionnaire » ou de "pionnier » qui se bat dans un but plus noble (car collectif) que le simple gain d'argent. Il s'agit d'un mode de présentation de soi particulièrement flatteur : le commerçant, qui mène ici sa " mission » en collaboration avec l'État grec, devient le protagoniste de cette épopée et refuse de 
distinguer entre intérêt privé, public ou national ${ }^{6}$. Paradoxalement, le discours de ce grossiste n'est pas différent de celui d'un tenancier d'un petit commerce qui peine à survivre : ce qui compte n'est pas le degré de réussite économique, mais sa traduction en fierté nationale?

La diffusion croissante de produits grecs à Bruxelles n'a pas été pourtant uniquement liée aux initiatives des commerçants grecs. Autre facteur à prendre en considération, l'internationalisation de la ville de Bruxelles qui débute en 1958, quand elle devient le centre des Institutions européennes ; les « Eurocrates » qui s'y installent contribuent, par leur demande, à la diversification du paysage alimentaire bruxellois. L'admission de la Grèce dans la Communauté européenne a également conduit à l'arrivée d'une nouvelle vague de Grecs. Ces «Eurocrates " grecs qui travaillent dans les Institutions européennes sont plus diplômés, fortunés et raffinés que les immigrés des années 1950 (souvent d'origine rurale). Ils constituent une clientèle exigeante et encouragent la création de commerces de haute gamme : leur rôle dans la visibilité accrue de la « civilisation grecque » dans la capitale européenne est considérable (Seraïdari, 2012a). De ce point de vue, le décloisonnement du domaine alimentaire à Bruxelles est lié tant aux initiatives des immigrés qui s'installent dans les anciens quartiers ouvriers qu'à la présence grandissante des « Eurocrates » dans les beaux quartiers : leur concentration est le résultat du développement institutionnel de l'Union européenne. Indiscutablement, l'internationalisation est aussi une affaire de classes sociales, dans la mesure où les circuits des produits de haute et de basse gamme ne se mélangent pas.

Il faut aussi prendre en compte le fait que certains produits grecs, comme la feta, sont progressivement devenus populaires grâce à une tendance internationale de diversification alimentaire qui se développe après la Deuxième Guerre mondiale. Si les Belges poussent plus facilement la porte d'un commerce grec à Bruxelles grâce à la sortie du film Jamais le dimanche en 1960 et le succès mondial du film Zorba le Grec réalisé en 1964, la Grèce devient également une destination touristique à la mode : suite à l'essor du tourisme de masse dans les années 1950-1960 après la généralisation des congés

\footnotetext{
${ }^{6}$ Un grand nombre d'immigrés grecs, surtout après la guerre civile (1946-1949), ont quitté leur pays pour des raisons politiques, puisqu'ils étaient catégorisés comme communistes. Ceux-ci n'auraient jamais tenu des discours de ce type, étant donné que la question des intérêts privés/intérêts nationaux ne se posait pas pour eux dans ces termes. Même si dans cet article je ne présente pas les récits de vie que j'ai recueillis, il convient de rappeler que le parcours de vie, les projets et les sensibilités politiques de ces personnes étaient très variés. ${ }^{7}$ Et cela, malgré le fait que beaucoup de produits proposés dans les épiceries grecques de Bruxelles étaient fabriqués dans d'autres pays européens que la Grèce. Ce qui signifie que ce n'est pas forcément l'État national qui profite économiquement de l'extension de la zone géographique de rayonnement de ses produits. C'est, en partie, pour pallier à ce problème que l'Appellation d'origine protégée (AOP) a été créée au sein de l'Union européenne.
} 
payés, les classes moyennes et populaires européennes découvrent et se familiarisent avec la cuisine grecque.

Il s'avère toutefois difficile de déterminer si c'est l'augmentation de l'offre (et de visibilité) ou la croissance de la demande qui est à l'origine de la popularité de certains produits, longtemps catégorisés comme ethniques. Entre l'expérience cinématographique $^{8}$, l'expérience touristique et l'expérience gustative dans les commerces grecs de Bruxelles, il est difficile de définir avec précision laquelle a joué le plus grand rôle. Ce qui signifie que le spectateur d'un film, le touriste et le commerçant immigré participent, chacun à sa manière, à cette « mondialisation par le bas » qui a connu une accélération notable après la Deuxième Guerre mondiale.

\section{L'internationalisation après la Deuxième Guerre mondiale}

L'internationalisation du secteur de l'alimentation entre dans une nouvelle phase tant en Belgique qu'en Australie après la Deuxième Guerre mondiale. Melbourne attire alors des migrants grecs qui ouvrent des restaurants proposant des spécialités grecques. Dans le même temps, cette ville accueille une vague d'immigration italienne qui joue un rôle important dans la transformation du paysage marchand : c'est là que le premier « espresso café » ouvre en 1954 ; Brown-May (2001) donne même à cette ville le titre de « capitale du café » en Australie. Melbourne lance ainsi la mode de l'espresso dans le pays. Dans les années 1950, Melbourne et Bruxelles semblent suivre une évolution parallèle, dans la mesure où l'arrivée de groupes immigrés de différentes origines bouscule progressivement les habitudes et favorise l'introduction de nouvelles pratiques. Ces acteurs de la « mondialisation par le bas », mais aussi les quartiers et, par extension, les villes dans lesquelles ils s'installent et développent leurs activités marchandes, peuvent alors se présenter a posteriori comme des "précurseurs ».

Si la période d'après la Deuxième Guerre mondiale est propice à cette évolution, c'est aussi parce que l'industrialisation de l'agroalimentaire et les techniques de conservation, de conditionnement et de transport, qui déconnectent pour la première fois l'aliment de son enracinement géographique et des contraintes climatiques, le permettent. L'internationalisation du secteur de l'alimentation est, dans une large mesure, le produit de la croissance des mouvements migratoires (et touristiques), mais elle est aussi

\footnotetext{
${ }^{8}$ La culture populaire de l'entertainment désigne une « forme de mondialisation culturelle » sur laquelle les États-Unis, à travers Hollywood, ont longtemps exercé une suprématie incontestable (Rocher, 2001 : 23). Mais l'américanisation ne constitue pas le seul effet de cette hégémonie culturelle : comme nous l'avons vu, l'industrie cinématographique américaine a pavé le chemin pour les activités marchandes des Grecs tant en Australie qu'en Belgique. Une « forme de mondialisation culturelle » peut donc avoir des effets économiques et déclencher des processus qui n'ont rien à voir avec les intentions de ceux qui l'ont initiée et développée.
} 
rendue possible par les avancées techniques assurant la circulation de produits standardisés et transportables. Entre 1950 et 2006, les exportations mondiales de marchandises ont été multipliées par près de 30 en volume : selon Rastoin et Ghersi, « [l'] internationalisation est un phénomène centrifuge qui se manifeste à travers une progression rapide des échanges depuis un demi-siècle » $(2010: 268)$. La généralisation de l'ouverture commerciale et l'expansion du volume des échanges sont constatées même par ceux qui adoptent une approche "internationale » et qui tiennent à nous rappeler que la mondialisation actuelle est très similaire à celle qui se dessinait avant la Première Guerre mondiale (Siroën, 2004).

La grande distribution fait aussi son apparition dans les années 1950 et devient un symbole de la modernité. L'entreprise Delhaize ouvre son premier supermarché en Belgique en 1957 : « la presse, y compris la jeune télévision, voit dans le supermarché comme un pas supplémentaire vers la modernité, qui rapproche un peu la vieille Europe des États-Unis » (Collet, 2003 : 97). Même discours quand l'hypermarché d'Auderghem est fondé le 15 septembre 1961 : « des hommes tournés vers l'avenir [...] viennent ainsi de parachuter à $5 \mathrm{~km}$ de la moyenâgeuse Grand-Place, un complexe de distribution comme on n'en avait jamais vu sur notre continent » (Grimmeau, 2013, paragraphe 12). L'innovation du supermarché, dont les Belges revendiquent la paternité en ce qui concerne son implantation sur le continent européen, devient ainsi un élément qui diminue la distance entre une « vieille Europe » (remplie de monuments « moyenâgeux ») et les États-Unis, pays considéré comme dynamique et pionnier. Alors que l'introduction des pratiques américaines dans le domaine de l'alimentation se fait en Belgique grâce à des entrepreneurs qui font des investissements importants, en Australie ce sont les immigrés grecs qui ont joué un rôle similaire, avec des moyens financiers bien plus limités, et cela bien avant la Deuxième Guerre mondiale.

\section{Immigrés grecs en Belgique et en Australie, des voies parallè̀les}

Aujourd'hui, le Café grec et le modèle de consommation auquel il a été associé en Australie sont autant en déclin que les épiceries tenues par des Grecs à Bruxelles. Elles non plus n'ont pu résister à la concurrence des supermarchés. Mais cela n'est pas le seul point commun. Dans les deux cas, le succès initial de ces commerces était basé sur la notion d'exotisme : les produits grecs vendus dans les commerces bruxellois paraissaient aussi exotiques aux yeux des clients belges que les nouveautés américaines proposées dans les Cafés grecs à la clientèle australienne. Cette idée est omniprésente dans l'ouvrage d'Alexakis et Janiszewski. Bien sûr, la notion d'exotisme ne renvoie pas à la même chose : dans le cas australien, c'est la modernité (américaine) qui est exotique ; dans le cas bruxellois, c'est l'authenticité des produits provenant d'un pays encore traditionnel et peu industrialisé (et dans le même temps, célèbre pour son héritage antique) qui est exotique. Après la Deuxième Guerre mondiale, cette quête d'exotisme 
pousse certains Cafés grecs à servir des plats chinois et italiens afin de satisfaire une nouvelle demande (Alexakis, Janiszewski, 2016 : 165). Un Café grec de Melbourne a même la réputation d'être le premier à introduire dans cette ville les spaghettis bolognaise (Ibid. : 173).

À travers la comparaison de ces deux trajectoires marchandes, nous constatons que l'internationalisation d'un commerce peut, d'une part, être le résultat d'une demande réduite, comme en Australie, où les commerçants grecs avaient choisi de gommer toute spécificité nationale renvoyant au pays d'origine. D'autre part, elle correspond à une perspective viable, comme cela a été le cas pour les commerçants grecs de Bruxelles qui ont décidé d'ouvrir, près des magasins tenus pas des immigrés d'autres origines, des boutiques vendant des produits grecs. Dans ce dernier cas, le déclin a obligé depuis quelques années les commerçants grecs à diversifier leur offre, qui tend à perdre complètement son caractère national. Si ces magasins sont devenus aussi « internationaux » que ceux que les Grecs tenaient jadis en Australie, c'est parce que ce qui faisait encore partie d'un exotisme « ethnique » dans les années 1960-1990 s'est progressivement transformé en consommation courante. En effet, il est difficile d'assigner actuellement une identité « nationale » à la feta ou au tarama. Une consommation courante et une production internationale, traversée par la rivalité entre différents pays producteurs, transforment inévitablement un produit national en produit international'.

Le déclin des épiceries grecques à Bruxelles et des Cafés grecs en Australie est, entre autres, le résultat d'un changement de modèle de consommation : les produits standardisés remplacent progressivement les spécialités « faites maison » dont les commerces en question avaient l'exclusivité. Tel est ce qui ressort du témoignage d'une épicière qui tient une boutique dans le quartier bruxellois de la Chasse depuis 1983 :

Avant, je faisais du tarama, maintenant je ne fais que du tzatziki. Les gens vont au supermarché, ils ne viennent pas ici pour acheter. [...] Les gens n'achètent pas non plus de fruits et de légumes chez nous, ils veulent des emballages, ils veulent que tout soit emballé [ta theloun paketarismena], comme dans le supermarché. (entretien, lundi 18 novembre 2013)

\footnotetext{
${ }^{9}$ Elia Petridou (2005) analyse les négociations juridiques et la concurrence marchande autour de la commercialisation de la feta, à qui l'AOP a été accordée par la Communauté européenne en 1996. Sur la manière dont la Grèce se sert de la référence à la Grèce antique pour assurer ses droits de propriété sur ses produits et son patrimoine culturel, tout en leur déniant un éventuel caractère balkanique, voir (Seraïdari, 2012b). Dans le cas de la feta, il s'agit d'un produit national (selon la position grecque qui a finalement reçu le soutien de l'Union Européenne) qui a été présentée par des concurrents commerciaux européens comme étant un produit balkanique. Ce produit peut donc être défini selon quatre échelles : locale (selon les lieux de production grecs), nationale (grâce à la reconnaissance juridique de l'AOP), régionale (en référence au caractère balkanique qui lui a été attribué par certains concurrents au sein du marché européen) et internationale (à cause d'une consommation et d'une production généralisée).
} 
Lors de mes enquêtes de terrain, j'ai assisté à une scène surprenante dans une épicerie grecque de Woluwe-Saint-Pierre (jeudi 17 octobre 2013). Un client belge, qui de toute évidence n'est pas un habitué, cherche un fromage grec, le manouri, pour essayer une nouvelle recette. Il explique aux commerçants grecs qu'il est certain de pouvoir le trouver au Delhaize, mais il voudrait d'abord s'assurer qu'il ne peut pas trouver une forme plus artisanale de ce fromage dans leur épicerie. Les épiciers sont embarrassés, parce qu'ils proposent dans leur magasin une toute petite gamme de fromages grecs qui, de plus, sont tous de provenance industrielle. Ils n'ont pas de manouri, en fait ils ne savent même pas précisément à quel type de fromage ce nom correspond. À la fin de la conversation, le client repart pour aller acheter le produit qui lui manque au Delhaize. Cet échange économique « raté » entre un commerçant et un acheteur potentiel révèle que le premier n'est pas toujours l'expert que le second s'attend à rencontrer. Dans ce cas, le client découvre non seulement la banalité de l'offre des fromages grecs dans ce lieu qui est censé être spécialisé, mais aussi le manque de savoir des commerçants : ceux-ci non seulement ne vendent pas ce type de fromage, mais ne l'utilisent pas non plus pour faire la cuisine chez eux. Cette scène montre également la distance parcourue depuis le temps des " pionniers », c'est-à-dire depuis la période où les commerçants grecs voulaient faire connaître aux Belges des produits qui ne leur étaient pas encore familiers. Selon une autre épicière qui tient un magasin à Schaerbeek depuis 1969 :

À l'époque, il n'y avait que des produits belges, on ne trouvait pas de produits grecs à manger, même pour nous. Nous avons été les premiers à faire venir des produits grecs. Au début, on les donnait aux Belges gratuitement, pour qu'ils goûtent, et ils ne voulaient même pas goûter. Mais petit à petit, ils les ont appris, la feta, le tarama, les dolmas. Maintenant ils les connaissent mieux que nous. (entretien, jeudi 13 juin 2013)

Une fois que les frontières entre le caractère national et international des marchandises sont brouillées, la notion même de spécificité nationale perd sa pertinence. En effet, dès qu'un produit devient trop familier, il faut le repositionner dans le champ marchand qui manie, à son gré, les notions de proximité et de distance : une des solutions possibles est de promouvoir une variante plus distante, moins connue et donc, plus désirable. La quête du manouri à laquelle j’ai assisté révèle ce processus.

\section{En guise de conclusion}

Pour les Grecs de Belgique, la dynamique d'internationalisation n'a pas été jugée contradictoire avec le maintien des modèles de consommation du pays d'origine. Bien au contraire, c'est en rendant public et accessible ce que les Grecs d'Australie appelaient « notre nourriture secrète » que les commerces grecs de Bruxelles ont prospéré - au moins, jusqu’à leur déclin récent qui a changé la donne. Ce qui pour les Grecs d’Australie constituait une barrière à ne pas franchir a longtemps été pour les Grecs de Bruxelles un atout commercial qui les érigeaient en connaisseurs " innés », et leur assuraient même un certain monopole - la banalisation des produits jadis considérés 
comme " exotiques » mettant fin à ce privilège commercial. Si les Grecs de Bruxelles traduisent souvent leur activité commerciale en fierté nationale, la forme d'ethnicisation que les Grecs introduisent dans le paysage alimentaire australien jusque dans les années 1970 n'est pas liée à leur pays d'origine, mais aux États-Unis. Dans les deux cas, l'orientation de leurs activités et le choix du domaine de l'ethnicisation (modèle de consommation américain pour les uns, et grec pour les autres) dépendent, dans une large mesure, des signaux donnés par le marché. Ils sont donc plus pragmatiques qu'idéologiques.

Nous pouvons ainsi distinguer entre deux formes d'internationalisation : une forme qui s'appuie sur la spécificité nationale, et une autre qui la refuse. Autrement dit, une forme qui maintient les barrières nationales et une autre qui tend à les abolir. De ce point de vue, la première configuration s'appuie sur l'exoticisation, soit de la modernité, en ce qui concerne les produits américains en Australie, soit de la tradition, dans le cas des pratiques culinaires grecques à Bruxelles. La deuxième, en revanche, neutralise l'exotisme, soit par l'absence de demande et d'ouverture, comme dans le cas de l'Australie avant la Deuxième Guerre mondiale où les commerçants grecs n'ont même pas essayé d'imposer leurs propres pratiques culinaires, soit par la banalisation actuelle des produits qui tendent à perdre leur caractère national. Si la première configuration a permis aux commerçants grecs d'être des vecteurs de changement, la seconde s'avère neutralisante. Dans le cas de la banalisation, cette forme d'internationalisation semble même conduire à une impasse : il n'y a plus de terra incognita, il n'y a plus de produits inconnus à populariser. Seule sortie de secours, le passage de l'ethnicisation et de l'espace national à d'autres schémas de territorialité : il faut contourner le national (lié de plus en plus à des produits standardisés) en faveur du local, du « bio » et du terroir, présentés dorénavant comme des réservoirs encore inexplorés d'authenticité (Seraïdari, 2016). La mondialisation sait délocaliser, mais aussi relocaliser.

La banalisation n'est donc qu'une étape intermédiaire, suivie par une segmentation qui vise à renouveler les expériences et à résister à la saturation. Le besoin de sortir de l'impasse de la banalisation assigne un rôle nouveau aux États nationaux qui appuient l'institutionnalisation des AOP (et des autres labels de ce type) et qui régulent, par des contrôles et des mesures législatives, le développement du « bio ». Ces mutations - qui concernent tant la redéfinition des marchés et des produits que le changement des mentalités de ceux qui fabriquent, vendent et consomment ces produits - révèlent les nouveaux chemins que l'internationalisation du goût emprunte aujourd'hui.

\section{Bibliographie}

AlEXAKIS E., JANISZEWSKI L. (2016), Greek cafés and milk bars of Australia, Canberra, Halstead Press.

Brown-May A. (2001), Espresso! Melbourne Coffee Stories, Melbourne, Arcadia. 
COllEt E. (dir.) (2003), Delhaize « Le lion». Épiciers depuis 1867, Tournai, Delhaize group.

DebOUVERIE A. (1992), Visages économiques d'Obbrussel. Saint-Gilles d'hier et d'aujourd'hui, Bruxelles, Syndicat d'initiative de Saint-Gilles.

GRIMMEAU J.-P. (2013), « Un anniversaire oublié : les premiers hypermarchés européens ouvrent à Bruxelles en 1961 ", Brussels Studies, n 67. En ligne, consulté le 1 février 2015. URL : https://journals.openedition.org/brussels/1155

MARCIHAC V. (2012), Le luxe alimentaire. Une singularité française, Rennes, Presses universitaires de Rennes/Presses universitaires François-Rabelais de Tours.

PAPAgeorgiou A. (2010), " Perceptions et choix des consommateurs grecs face à la mondialisation de la grande distribution alimentaire », Économie rurale, $\mathrm{n}^{\circ} 318-319$, p. 50-64.

Petridou E. (2005), « Au pays de la feta. Négociation de la grécité dans le contexte européen », Ethnologie française, $\mathrm{n}^{\circ}$ 2, vol. 35, p. 255-265.

Portes A. (1999), « La mondialisation par le bas. L'émergence des communautés transnationales », Actes de la recherche en sciences sociales, vol. 129, n 1, p. 15-25.

RAStOIN J.-L., Ghersi G. (2010), Le système alimentaire mondial. Concepts et méthodes, analyses et dynamiques, Versailles, Editions Quæ.

ROCHER G. (2001), « La mondialisation : un phénomène pluriel ? », in D. Mercure (dir.), Une société monde? Les dynamiques sociales de la mondialisation, Sainte-Foy, Les Presses de l'Université Laval, p. 17-31.

SERAÏDARI K. (2012a), La ville, la nation et l'immigré. Rapports entre Grecs et Turcs à Bruxelles, Paris, L'Harmattan.

SERAÏDARI K. (2012b), " L'Homo Balkanicus en contexte migratoire. Un modèle d'identification à défendre ou à rejeter ? ", Civilisations, vol. 60, nº 2, p.103-118.

SERAÏDARI K. (2016), « Exotisme, initiatives marchandes et pratiques alimentaires dans les commerces grecs de Bruxelles ", Cahiers balkaniques - Hors série. En ligne, consulté le 3 avril 2016. URL : http//ceb.revues.org/7115

SiroËN, J.-M. (2004), « L'international n'est pas le global. Pour un usage raisonné du concept de globalisation », Revue d'économie politique, vol. 114, nº 6, p. 681-698. 